\title{
Improving Forecast Skill by Assimilation of Quality Controlled AIRS Version 5 Temperature Soundings
}

\author{
Joel Susskind ${ }^{1}$ and Oreste Reale ${ }^{2}$ \\ ${ }^{1}$ NASA Goddard Space Flight Center, Greenbelt, MD, USA 20771 \\ ${ }^{2}$ UMBC, NASA Goddard Space Flight Center, Greenbelt, MD, USA 20771
}

\begin{abstract}
The AIRS Science Team Version 5 retrieval algorithm has been finalized and is now operational at the Goddard DAAC in the processing (and reprocessing) of all AIRS data. The AIRS Science Team Version 5 retrieval algorithm contains two significant improvements over Version 4: 1) Improved physics allows for use of AIRS observations in the entire $4.3 \mu \mathrm{m} \mathrm{CO}_{2}$ absorption band in the retrieval of temperature profile $T(p)$ during both day and night. Tropospheric sounding $15 \mu \mathrm{m} \mathrm{CO} \mathrm{CO}_{2}$ observations are now used primarily in the generation of cloud cleared radiances $\hat{R}_{i}$. This approach allows for the generation of accurate values of $\hat{R}_{i}$ and $T(p)$ under most cloud conditions. 2) Another very significant improvement in Version 5 is the ability to generate accurate case-by-case, level-by-level error estimates for the atmospheric temperature profile, as well as for channel-by-channel error estimates for $\hat{R}_{i}$. These error estimates are used for Quality Control of the retrieved products.
\end{abstract}

We have conducted forecast impact experiments assimilating AIRS temperature profiles with different levels of Quality Control using the NASA GEOS-5 data assimilation system. Assimilation of Quality Controlled $T(p)$ resulted in significantly improved forecast skill compared to that obtained from analyses obtained when all data used operationally by NCEP, except for AIRS data, is assimilated. We also conducted an experiment assimilating AIRS radiances uncontaminated by clouds, as done operationally by ECMWF and NCEP. Forecast resulting from assimilated AIRS radiances were of poorer quality than those obtained assimilating AIRS temperatures.

Keywords: High spectral resolution IR sounders, atmospheric sounding, satellite meteorology, new theoretical developments, improved forecast skill, data assimilation, NWP, AIRS/AMSU, Quality Control, temperature profile

\section{INTRODUCTION}

AIRS was launched on EOS Aqua on May 4, 2002, together with AMSU-A and HSB, to form a next generation polar orbiting infrared and microwave atmospheric sounding system. ${ }^{1}$ The primary products of AIRS/AMSU-A are twice daily global fields of atmospheric temperature-humidity profiles, ozone profiles, sea/land surface skin temperature, and cloud related parameters including OLR. Also included are the clear column radiances $\hat{R}_{i}$ used to derive these products which are representative of the radiances AIRS would have seen if there were no clouds in the field of view. All products also have error estimates. The sounding goals of AIRS are to produce $1 \mathrm{~km}$ tropospheric layer mean temperatures with an rms error of $1 \mathrm{~K}$, and layer precipitable water with an rms error of 20 percent, in cases with up to 90 percent effective cloud cover. The products are designed for data assimilation purposes for the improvement of numerical weather prediction, as well as for the study of climate and meteorological processes. With regard to data assimilation, one can use either the products themselves or the clear column radiances from which the products were derived.

The basic theory used to analyze AIRS/AMSU/HSB data in the presence of clouds, called the at-launch algorithm, and that used in the Version 4 post-launch algorithm, which differed only in the minor details from the at-launch algorithm, has been described previously ${ }^{2,3}$. Susskind, et al. ${ }^{4}$ describe the AIRS Version 5 retrieval algorithm, which is now being used operationally at the Goddard DISC in the routine generation of geophysical parameters derived from AIRS/AMSU data. A major innovation in Version 5 is the ability to generate case-by-case level-by-level error estimates for retrieved 
quantities and the use of these error estimates for Quality Control. This paper will describe the use of Version 5 temperature profile error estimates $\delta T(p)$, for $T(p)$ Quality Control. The spatial coverage and accuracy of retrievals using different error estimate thresholds for Quality Control will be shown, as well as results of forecast impact studies assimilating AIRS Version 5 temperature profiles using different Quality Control thresholds.

\section{VERSION 5 ERROR ESTIMATES AND QUALITY CONTROL}

Coupled AIRS/AMSU-A retrievals in the presence of broken cloud cover are usually highly accurate. Under some conditions, such as complete overcast, combined AIRS/AMSU-A retrievals cannot be performed at all. In cases of complex clouds or terrain, retrievals are of poorer quality. In the pre-launch version of the AIRS/AMSU-A retrieval algorithm, Quality Control was applied uniformly to the entire profile. If any geophysical parameter was considered to be of poor quality, the whole set of retrieval geophysical parameters was rejected and clouds were derived using the MW state of Step (1) above. This "one size fits all" approach led to significant compromises between desired spatial coverage of accepted retrievals and desired accuracy. In Version $4^{3}$, the combined IR/MW retrieval parameters were retained, and used to derive cloud parameters, as long as it was felt that the combined IR/MW retrieval was at least as accurate as a MW only retrieval. This was considered to be true if the retrieved cloud fraction derived using the IR/MW state was less than or equal to $90 \%$ and an initial cloud clearing step was stable. If this test was passed (referred to as the Stratospheric Temperature Test), the temperature profile above $200 \mathrm{mb}$ was considered acceptable. Constituent profiles $\left(\mathrm{H}_{2} \mathrm{O}, \mathrm{O}_{3}, \mathrm{CO}\right.$, and $\mathrm{CH}_{4}$ ) were accepted if the Stratospheric Temperature Test was passed and additional slightly more stringent cloud clearing stability tests were also passed. The next level of test was applied to the temperature profile beneath $200 \mathrm{mb}$ and above $3 \mathrm{~km}$ (the Mid Tropospheric Temperature Test). Finally, a more stringent test was applied to accept temperature profiles in the lowest $3 \mathrm{~km}$ of the atmosphere (Lower Tropospheric Temperature Test). Lower tropospheric temperatures are the most difficult to determine accurately, both because of effects of low clouds on the radiances and uncertainty and small scale variability in surface skin temperature and emissivity. Both concerns create greater problems over land than ocean. In response to this, the Lower Tropospheric Temperature Test rejected lower tropospheric temperature more often over land than over ocean in Version 4.

The Version 4 Quality Control tests each used thresholds for values of 12 different parameters $Y_{k}(k=1,12)$ representative of residuals of internal convergence tests ${ }^{3}$. In Version 5 , the case-by-case values of each of the parameters whose thresholds were used in the Version 4 acceptance tests, $Y_{k}$, are used in the generation of error estimates of the individual retrieved parameters. Values of four other retrieval convergence tests are included as well, giving a total of 16 tests. In the case of either $T(p), T_{\text {skin }}$, or $W_{t o t}$ (total precipitable water), $\delta X_{i}$, the error estimate of geophysical parameter, $X_{i}$, is expressed according to

$$
\delta X_{i}=\sum_{k=1}^{16} M_{i k} Y_{k}
$$

where $Y_{k}$ is the value of the $k^{\text {th }}$ test, and $M$ is a matrix with different values over ocean and land. Susskind et al. ${ }^{4}$ give more details about the term $Y_{k}$ and the generation of $M$. Error estimates are, by definition, all positive.

\subsection{Version 5 Temperature Profile Quality Control}

Case-by-case level-by-level error estimates for temperature profiles at pressure $p_{i}$ are obtained by equation 1 using the appropriate values of $\mathrm{Y}_{\mathrm{k}}$ for each profile. These error estimates are used to determine a case-by-case characteristic pressure $p_{\text {best }}$, down to which the profile is considered acceptable for data assimilation purposes. All accepted profiles are assigned to have high quality down to at least $70 \mathrm{mb}$. The characteristic pressure $p_{\text {best }}$ is defined as the highest pressure (somewhere between $70 \mathrm{mb}$ and $p_{\text {surf }}$ ) at which the error estimate in each of the next 3 pressure levels is not greater than a pressure dependent error estimate threshold.

Pressure dependent thresholds are determined from a set of 3 threshold parameters $\delta T_{70}, \delta T_{\text {mid }}$, and $\delta T_{\text {surf }}$ representative of error thresholds for $T(p)$ at $p=70 \mathrm{mb}$, at $p=p_{\text {surf } / 2}$, and at $p=p_{\text {surf }}$ where $p_{\text {surf }}$ is the surface pressure. The thresholds $\delta T_{i}$ at intermediate pressures are linearly interpolated in $\log p$ between the given values. It was found advantageous to have separate error thresholds for non-frozen ocean on the one hand, and land and ice on the 
other. Table 1 shows the Standard Version 5 thresholds for both non-frozen ocean (called ocean) and other than nonfrozen ocean (called land). These thresholds are the ones used at the Goddard DISC in the Version 5 Quality Control, and were optimized to provide reasonable spatial coverage for both data assimilation and climate purposes. Subsequent research has shown that tighter Quality Control performs better for data assimilation proposes, while looser Quality Control (better spatial coverage) performs better for climate purposes. Table 1 also shows values of two other sets of thresholds that we have used for data assimilation experiments. These are more stringent sets of thresholds than the Standard version 5 thresholds and are labeled Medium and Tight.

Table 1

Temperature Profile Thresholds (K)

\begin{tabular}{lcccccc} 
& \multicolumn{3}{c}{ Ocean } & \multicolumn{3}{c}{ Land } \\
& $\delta T_{70}$ & $\delta T_{\text {mid }}$ & $\delta T_{\text {surf }}$ & $\delta T_{70}$ & $\delta T_{\text {mid }}$ & $\delta T_{\text {surf }}$ \\
Standard & 1.75 & 1.25 & 2.25 & 2.25 & 2.0 & 2.0 \\
Medium & 1.75 & 1.0 & 1.75 & 1.75 & 1.0 & 2.0 \\
Tight & 1.75 & 0.75 & 1.75 & 1.75 & 0.75 & 1.75
\end{tabular}

Figure 1a shows the rms error of global Quality Controlled temperature profiles for Version 4 (black) and Version 5 (gray) using the standard Version 5 thresholds for both land and ocean. Figure $1 \mathrm{~b}$ shows the percent of cases accepted for both Version 4 and for Version 5 using the Standard thresholds. The global percent accepted cases for Version 5 Standard is significantly higher than that of Version 4 at $300 \mathrm{mb}$ with a comparable accuracy. The same is true at all levels of the troposphere. The percent accepted above $200 \mathrm{mb}$ is somewhat lower in Version 5 Standard than in Version 4, with a significant improvement in sounding accuracy between $100 \mathrm{mb}$ and $200 \mathrm{mb}$. This is because not all cases called "Stratosphere Good" in Version 4 have sufficient accuracy down to $200 \mathrm{mb}$. The red and purple curves represent Quality Controlled Version 5 retrievals using the two sets of Medium and Tight thresholds as shown in the figure. They show how tightening thresholds can lead to significantly more accurate Quality Controlled retrievals, with a corresponding lower percentage of accepted retrievals, resulting in poorer spatial coverage. Figure 1 also contains analogous curves for Version 5 retrievals passing the AIRS essentially clear test (yellow). ${ }^{2}$ The essentially clear test allows for cases with up to $2 \%$ fractional cloud cover. These are slightly more accurate than those passing the Tight test, but represent only $10 \%$ of the cases.

Figure 2a shows the field of accepted $700 \mathrm{mb}$ temperature for ascending (daytime) orbits on January 25, 2003, with Version 5 Standard Quality Control. Areas shown in gray represent missing data. This is a result either of orbit gaps, a missing granule (over Central Africa), rejected soundings, or elevated terrain in which the surface pressure is lower than $700 \mathrm{mb}$. Version 5 soundings with Standard Quality Control have very good spatial coverage at $700 \mathrm{mb}$, and the thermal features are well defined and well represented. This good spatial coverage is needed for the purpose of generating climate data sets with a minimal clear sky bias. Very good spatial coverage is also potentially desirable for data assimilation purposes, provided the accuracy of the accepted retrievals is acceptable. Figure $2 \mathrm{~b}$ shows the spatial coverage of accepted Version 4 retrievals at $700 \mathrm{mb}$. The Version $4700 \mathrm{mb}$ spatial coverage is reasonably good, but significantly poorer than Version 5 Standard, particularly at high latitudes. Figures $2 \mathrm{c}$ and $2 \mathrm{~d}$ show spatial coverage for Version 5 with Medium and Tight thresholds. Version 5 Medium has somewhat better spatial coverage than Version 4, and Version 5 Tight has comparable spatial coverage to Version 4 at $700 \mathrm{mb}$. Areas with surface pressure less than 700 $\mathrm{mb}$, such as over East-Antarctica, show up as data gaps in this figure. 
LAYER MEAN RMS TEMPERATURE ("C)

GLOBAL DIFFERENCES FROM "TRUTH" January 25,2003 iobal

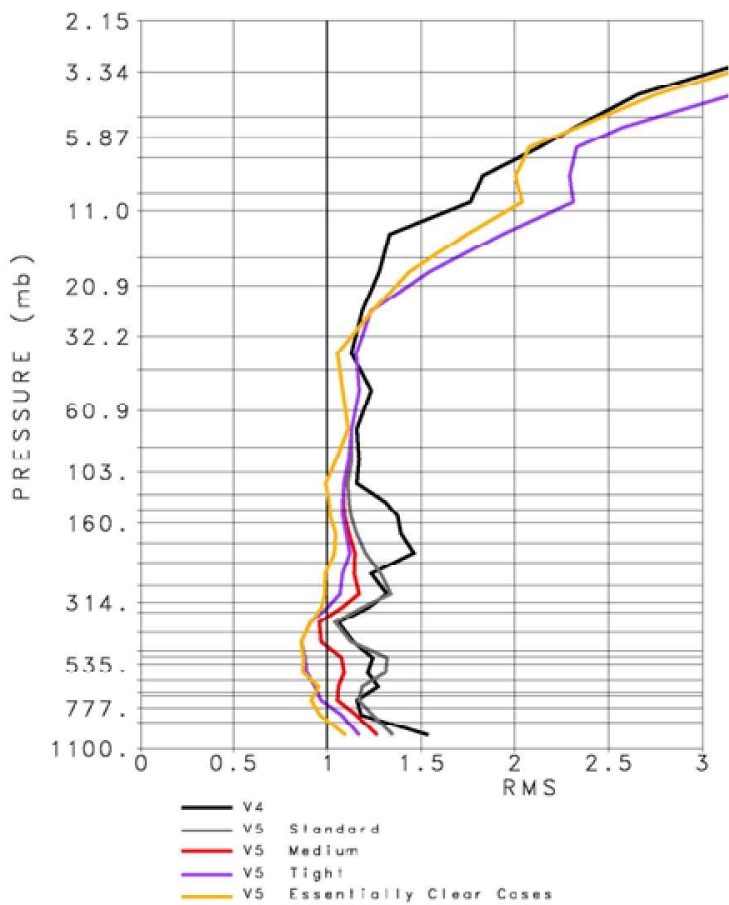

Figure 1a

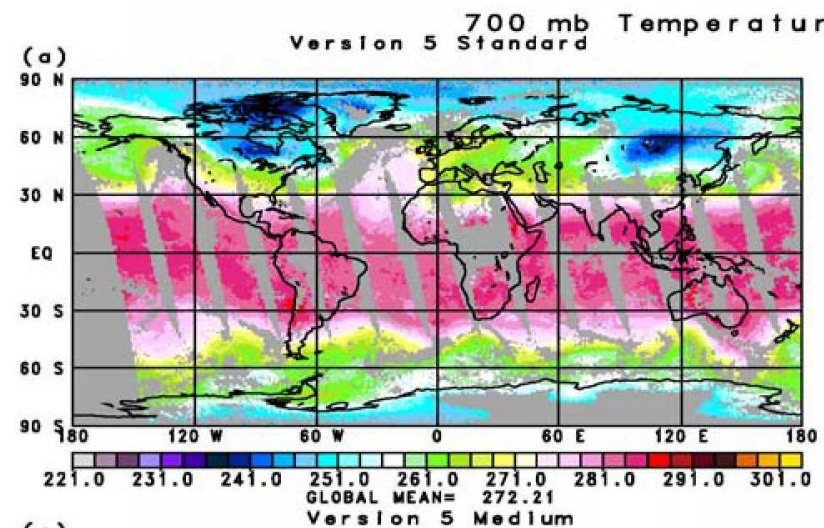

(2) Vorsion 5 Madium

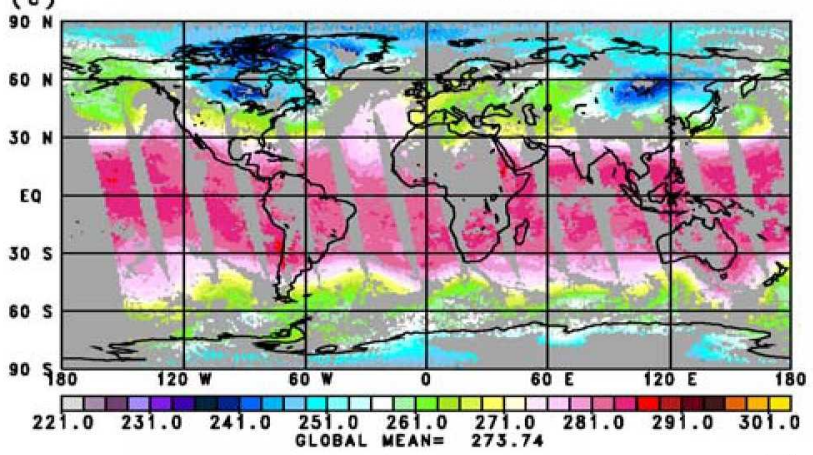

Percent of L R/MW Cases Included

January 25,2003

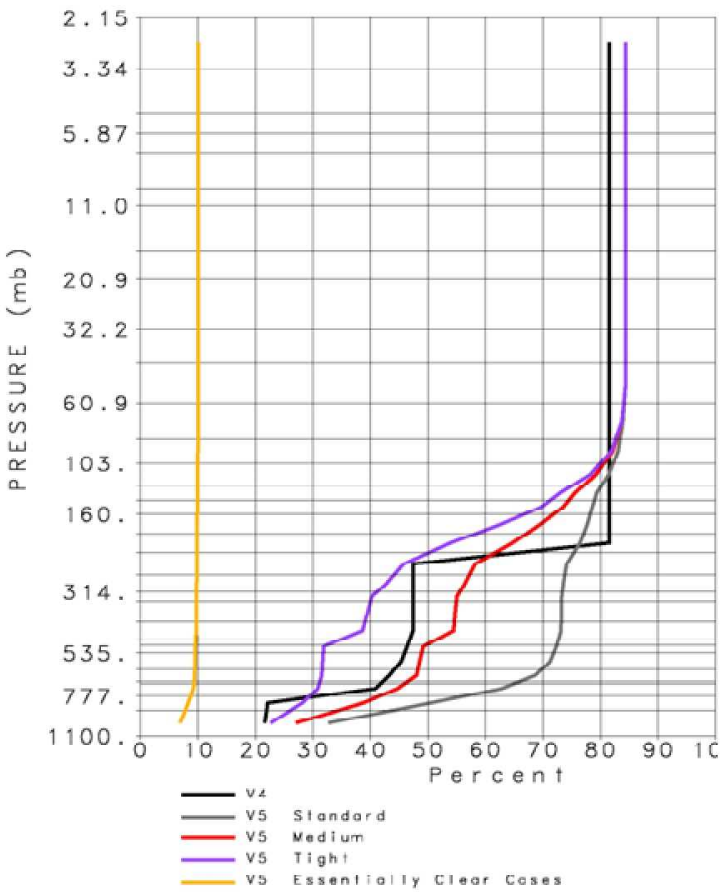

Figure 1b
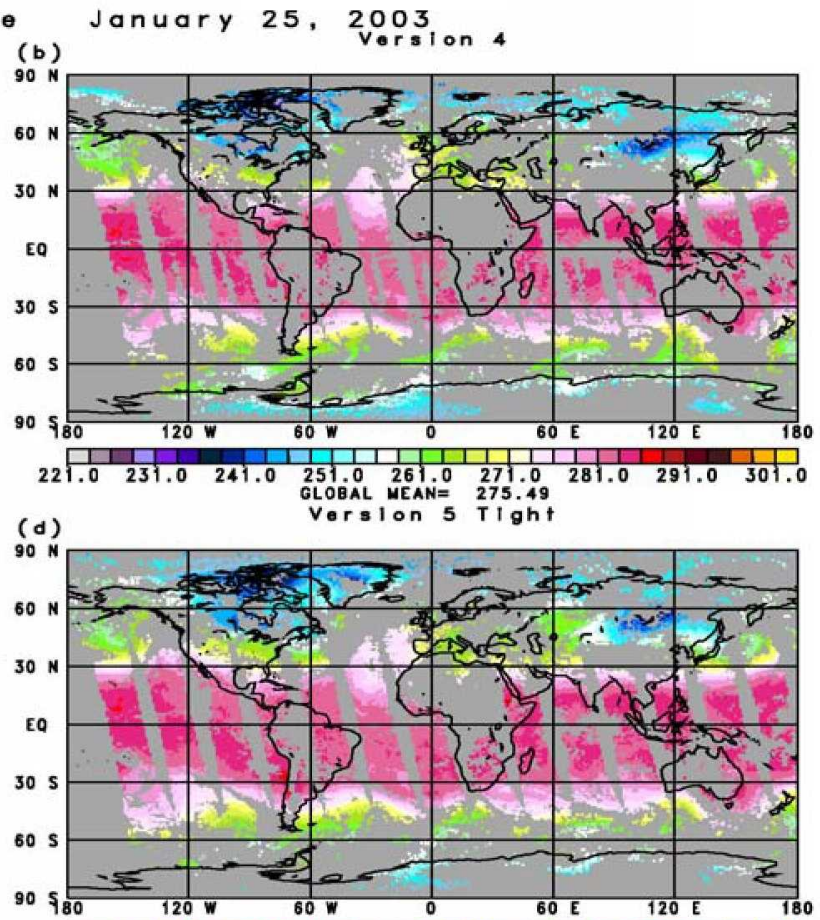

Figure 2 


\section{FORECAST IMPACT EXPERIMENTS}

Data from AIRS are becoming increasingly important in operational weather forecasting and have been proven beneficial in identifying the fine thermal structure of the atmosphere in a number of studies. Among these, Tian et al. ${ }^{5}$ demonstrate that AIRS-derived products improve the representation of the vertical structure of the tropical atmosphere, Le Marshall et al. ${ }^{6}$ show positive impact on the NCEP operation system's forecasting skill resulting from the assimilation of AIRS clearsky radiances, and $\mathrm{Wu}$ et al. ${ }^{7}$ show improvement in hurricane simulations by assimilating retrieved AIRS temperature and humidity profiles derived in clear conditions. However, the data used by these researchers are only in regions unaffected by clouds. This is a limitation in the optimal use of AIRS observations because of discontinuous coverage and because cloudy regions are likely to be more dynamically active.

We have conducted a number of data assimilation experiments as a step toward finding an optimum balance of spatial coverage and sounding accuracy with regard to improving forecast skill. The global data assimilation and forecasting system used is the NASA GEOS-5, which combines the Gridpoint Statistical Interpolation (GSI) analysis algorithm codeveloped by the National Centers for Environmental Predictions (NCEP) [e.g., Wu et al. ${ }^{8}$ ], with the NASA atmospheric global forecast model, documented by Bosilovich et al. ${ }^{9}$. The forecast model shares the same dynamical core $\operatorname{Lin}^{10}$ with the so-called finite-volume General Circulation Model (fvGCM), which has performed very well in studies focused on tropical cyclones [e.g. Atlas et al. ${ }^{11}$, Shen et al. ${ }^{12}$ ]. The GEOS-5 however contains a more recent version of the fvGCM, with a different set of physical parameterizations, partly developed by the NASA Global Modeling and Assimilation Office (GMAO). The model is run at a horizontal resolution of $0.5^{\circ}$ latitude $\times 0.67^{\circ}$ longitude with 72 vertical levels.

\subsection{Experiments During the Northern Hemisphere Winter Period January 1 to January 31, 2003}

The first set of experiments we conducted used data for the period January 1 to January 31, 2003. Seven day forecasts were run every two days beginning January 6, 2003, and forecasts every 12 hours were verified against the NCEP analysis, which was taken as "truth". In a first attempt to assess the tradeoff between spatial coverage and accuracy of soundings with regard to data assimilation, we assimilated Quality Controlled AIRS Version 5 retrieved temperatures down to $p_{\text {best }}$ using the three different Quality Control thresholds shown in Section 2.1. The AIRS Version 5 temperature profiles were presented to the GSI analysis as rawinsonde profiles, and the profile error estimates $\delta T(p)$ were used as the uncertainty for each measurement in the data assimilation process.

Forecasts generated from these three sets of analyses are compared to those from a "Control" analysis, in which all the data used operationally by NCEP at that time was assimilated, but no AIRS data was assimilated. Radiances from the Aqua AMSU-A instrument were assimilated operationally by NCEP and are included in the "Control". It should be noted that the Aqua orbit (1:30 ascending) is almost identical to that of NOAA 16 carrying HIRS3, AMSU-A and AMSU$B$, so AIRS/AMSU temperature soundings are providing additional information to that contained in the AMSUA/AMSU-B radiances on NOAA 16 in the same orbit, as well as those of the Aqua AMSU-A radiances. No AIRS data was assimilated operationally at that time.

Figure 3 shows the average of the 2712 hour to seven day forecast sea level pressure (SLP) anomaly correlation coefficients verified against the NCEP analysis for both Northern Hemisphere Extra-Tropics and Southern Hemisphere Extra-Tropics for all these experiments. The forecast results shown were made during the period January 5 to January 31, 2003. This allowed for a 5 day spin-up period at the start of the analyses. The higher the anomaly correlation coefficient, the more skillful the forecast is. An anomaly correlation coefficient of 1 represents a perfect forecast and a value of 0.6 is considered to be the lower boundary of a skillful forecast. In the Northern Hemisphere, assimilating AIRS soundings resulted in an improvement in average five day forecast skill of roughly five hours for all three sets of AIRS assimilations. At seven days, the forecast from the Tight assimilation maintained this improvement in skill, while the improvement dropped off slightly with loosening the QC thresholds. In the Southern Hemisphere Extra-Tropics, the same relative forecast skill, resulting from the three AIRS assimilations, held. This time, however, the Tight assimilation maintained control forecast skill at 7 days, while addition of more sounding data actually degraded forecast skill as judged by SLP. Results shown for this set of experiments indicate that assimilation of Quality Controlled AIRS temperature soundings extending down to the surface significantly improves forecast skill in the Northern Hemisphere 

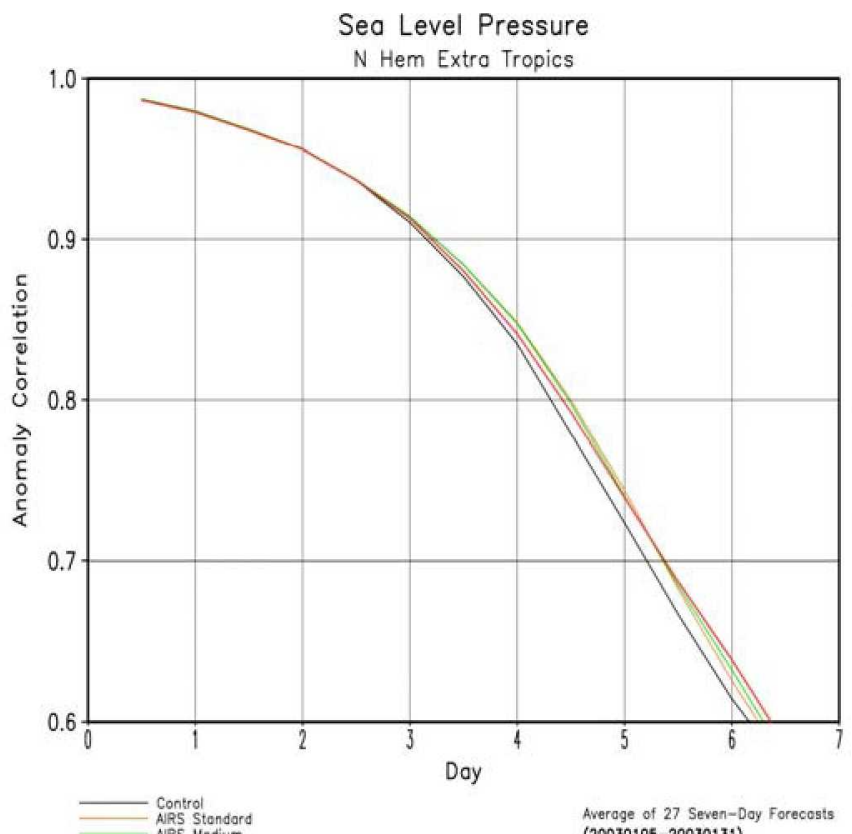
Ans Stondord
A ARS vegium
AlRS Tight

Figure 3a

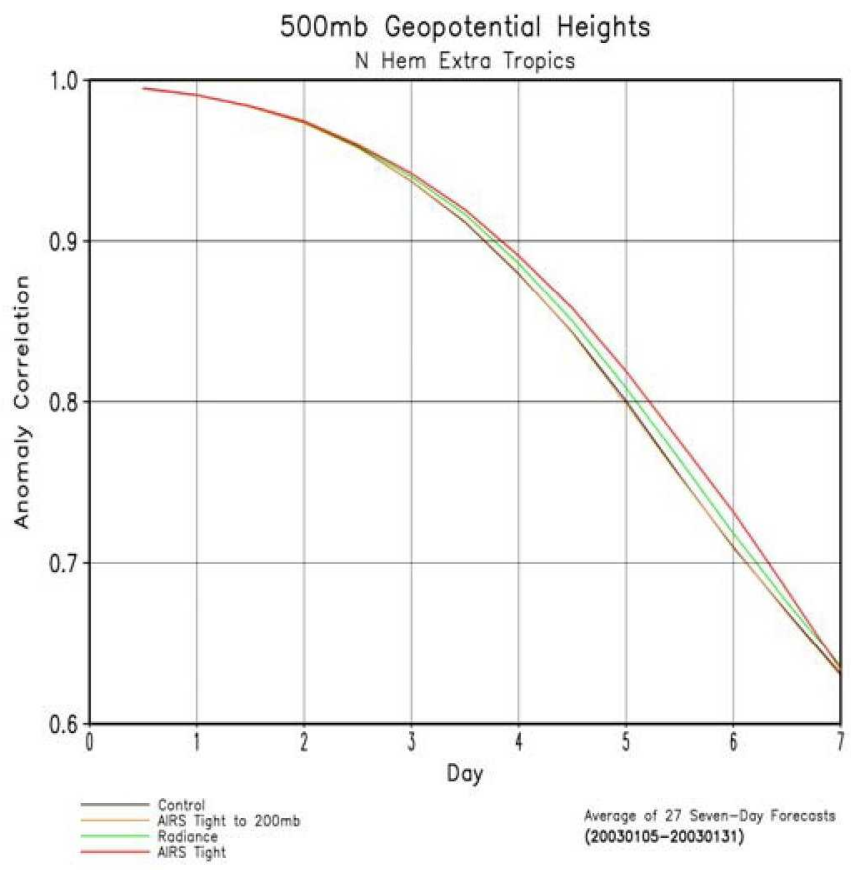

Figure 4a

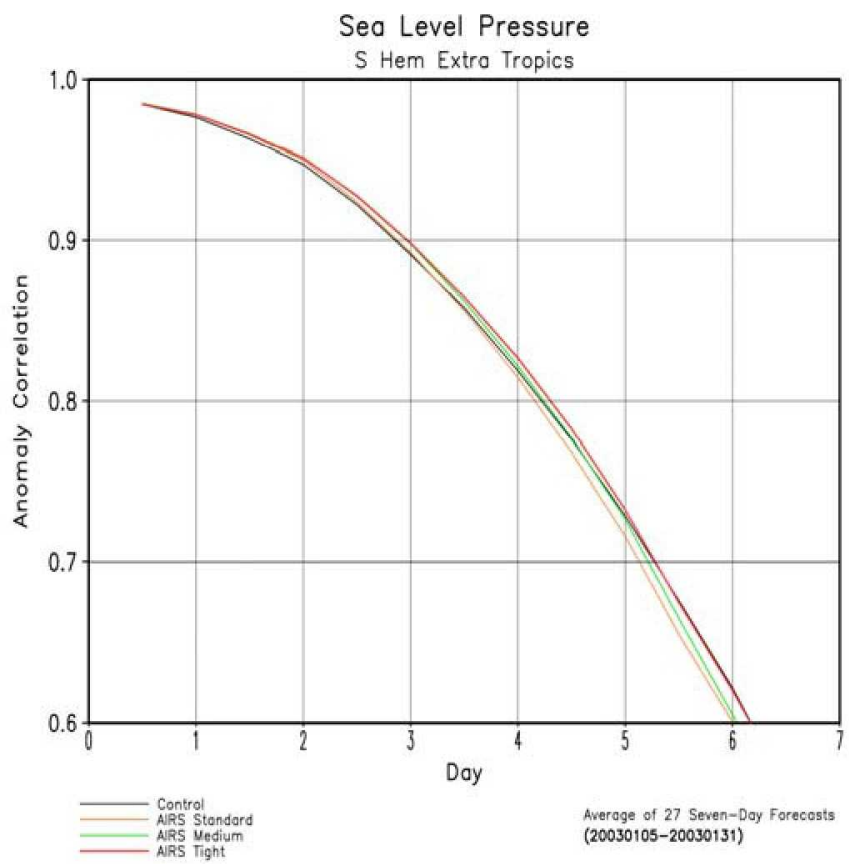

Figure 3b

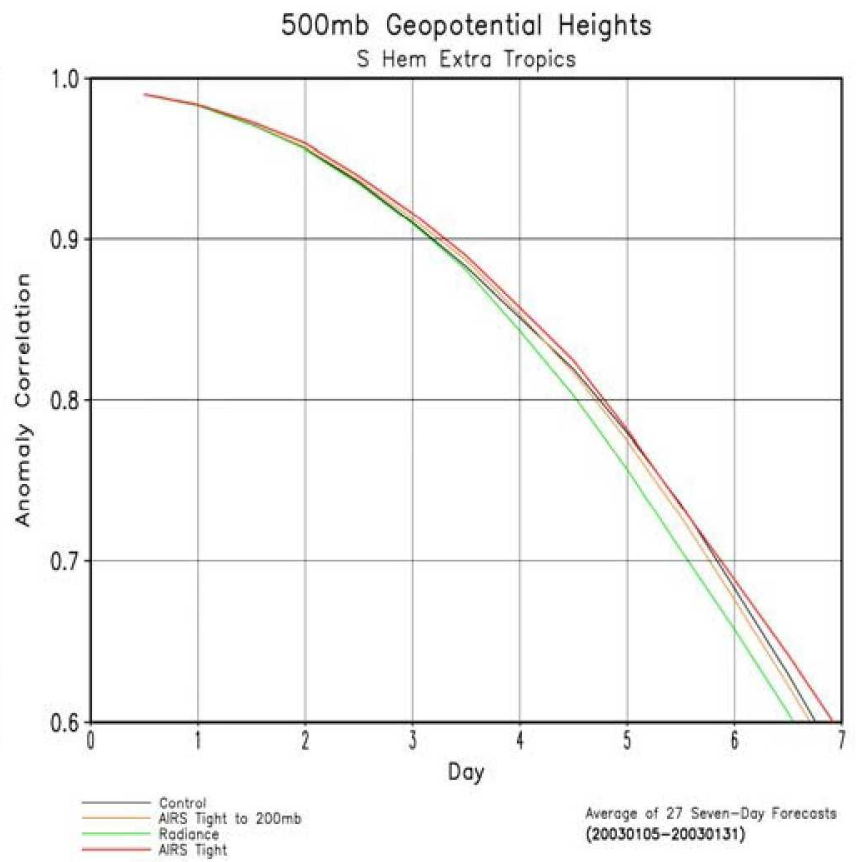

Figure 4b 
Extra-Tropics. Assimilation of soundings with Tight Quality Control performed better than with Medium or Standard Quality Control in both hemispheres. NCEP and ECMWF now assimilate AIRS observations operationally. The current operational practice is to directly assimilate observed AIRS radiances, rather than the AIRS temperature soundings as we did in the first set of experiments. The operational methodologies used by both NCEP and ECMWF do not have the capability to derive and assimilate cloud cleared AIRS radiances or to account for effects of clouds on the observed AIRS radiances. Instead, the analysis procedures used at both Centers select and assimilate only these AIRS observations which are "thought to be unaffected by clouds." These uncontaminated radiance observations are influenced primarily from temperatures in the stratosphere and also above clouds in areas where clouds are present. Results from AIRS retrievals indicate that roughly $95 \%$ of AIRS pixels are cloud contaminated. Therefore, information from most tropospheric sounding AIRS observations is not included in the operational AIRS radiance assimilation process.

We conducted a second set of experiments, also for the period January 1 to January 31, 2003, which were designed to assess the contribution of assimilation of AIRS tropospheric temperatures derived under primarily partially cloudy conditions toward the improvement of forecast skill in the midlatitudes. In the first of this set of experiments, we used the AIRS Tight Quality Control, but only assimilated those soundings down to at most $200 \mathrm{mb}$. In the second new experiment, we assimilated AIRS radiances according to the NCEP operational procedure. In these AIRS Radiance Assimilation experiments, all other data assimilated in the Control was also included, but no AIRS temperature profile data was assimilated.

Figure 4 shows analogous results to those shown in Figure 3, but for Northern Hemisphere and Southern Hemisphere Extra-Tropics $500 \mathrm{mb}$ Geopotential Height $\left(\mathrm{GPH}_{500}\right)$ anomaly coefficients. Results are shown for forecasts using the control (black) and AIRS Version 5 Tight (red) analyses as before. Also shown are results from the AIRS Version 5 Tight down to $200 \mathrm{mb}$ (orange) and AIRS Radiance Assimilation Analyses (green). Figure 4a shows the very significant result that virtually all the positive impact of assimilation of Quality Controlled AIRS temperature soundings in the Northern Hemisphere Extra-Tropics is lost if only AIRS stratospheric temperatures are used in the assimilation process (orange). The most important information is coming from tropospheric temperatures which are determined and assimilated primarily in partially cloudy scenes. In the Southern Hemisphere Extra-Tropics, the Tight QC assimilation actually improved 7 day forecast skill, as judged by the $\mathrm{GPH}_{500}$ anomaly correlation. This improvement was lost when only stratospheric soundings were assimilated.

Assimilation of AIRS radiances unaffected by clouds (green) resulted in a substantially reduced forecast impact in the Northern Hemisphere Extra-Tropics, and a significant negative forecast impact in the Southern Hemisphere ExtraTropics, compared to the assimilation of AIRS Tight Quality Controlled temperature profiles down to the surface. At least a part of this reduction in forecast impact of radiance assimilation in the Northern Hemisphere Extra-Tropics, results from the significant loss of spatial coverage of AIRS tropospheric sounding channels used in the data assimilation process due to cloud contamination. The cause of the substantial negative impact in the Southern Hemisphere ExtraTropics in the AIRS Radiance assimilation is unclear.

Reale et al. ${ }^{13}$ performed a case study looking at the difference between AIRS and Control Northern Hemisphere ExtraTropical analyses on January 25,2005 . This was a day on which 5 day forecasts from all analyses assimilating AIRS temperature soundings down to the surface performed significantly better than did that from the Control analysis. In particular, Reale et al. ${ }^{13}$ compared the Control analysis with the AIRS Medium analysis, and also with AIRS Medium down to $200 \mathrm{mb}$. The improvement in 5 day forecast skill obtained using the AIRS Medium analysis was traced to a significant difference in the boundary layer structure over the entire arctic region and parts of northeastern Siberia and Alaska as compared to what was found in the Control analysis. The AIRS Medium down to $200 \mathrm{mb}$ analysis differed insignificantly in the boundary layer from that of the Control in this area, and the 5 day forecast from that analysis showed no improvement over that of the Control. This confirms the finding that assimilation of Quality Controlled temperature in the lower troposphere, obtained under partial cloud cover, can significantly improve analyses and subsequent forecast skill. 


\subsection{Other Forecast Impact Experiments}

We have conducted two other sets of analogous data assimilation experiments during different seasons and in later time periods. The first set of experiments covered the time period October 15 to November 19, 2005 during Northern Hemisphere Fall. The second covered the time period April 15 to May 18, 2005 during the Northern Hemisphere Spring. In both sets of experiments, we conducted a Control assimilation, an AIRS Standard assimilation defining $p_{\text {best }}$ according to the AIRS Standard Quality Control thresholds, an AIRS Tight assimilation using AIRS Tight Quality Control thresholds, and an AIRS Radiance assimilation. These assimilations were all done in analogous manners to those performed in the January 2003 experiments.

Figures $5 \mathrm{a}$ and $5 \mathrm{~b}$ shows $500 \mathrm{mb}$ Geopotential Height anomaly correlation coefficients averaged over 36 seven day forecasts for the Northern Hemisphere and Southern Hemisphere Extra-Tropics respectively. In the Northern Hemisphere Extra-Tropics, 7 day forecasts from the AIRS Tight assimilation (red) had about an 8 hour improvement in forecast skill compared to the Control (black), while the Radiance assimilation (green) did not lead to improved forecast skill compared to the Control. Forecasts from the AIRS Standard assimilation resulted in an intermediate improvement in forecast skill. In the Southern Hemisphere Extra-Tropics, assimilation of AIRS temperature profiles using Tight Quality Control again resulted in the best forecast skill, closely followed by those resulting from the Radiance assimilation. At 5 days, forecasts resulting from the AIRS Standard assimilation provided a somewhat smaller positive impact compared to those from the Control. At seven days however, the assimilation of more, but somewhat less accurate, AIRS soundings resulted in a negative forecast impact compared to the Control. Findings from this set of experiments are for the most part consistent with those obtained from the January set of experiments.

Figures $6 \mathrm{a}$ and $6 \mathrm{~b}$ show results for analogous forecasts covering the period April 15 to May 18, 2008. Tropical Cyclone Nargis, which devastated parts of Myanmar on May 4, 2008, occurred during this time period. At the time of this writing, we have statistics only for forecasts out to 5 days, and these are what are presented in Figure 6, using a different vertical scale. As shown in Figures 3-5, improvement in 5 day forecast skill resulting from different assimilations is smaller than that in 7 day forecast skill, but can still be significant. Figure 6a shows a modest improvement in 5 day forecast skill in the Northern Hemisphere Extra-Tropics over this time period resulting from assimilation of AIRS temperatures with Tight Quality Control, which is not found in the forecasts resulting from the other assimilations. In the Southern Hemisphere Extra-Tropics, 5 day forecasts using AIRS Standard Quality Control had the largest positive impact compared to the Control, closely followed by forecasts using AIRS Tight Quality Control. Forecasts from the AIRS Radiance assimilation produced a smaller positive impact in this region compared to these from the Control.

All results shown thus far relate to the ability to produce better 5-7 day extra-tropical forecasts on the average. While this is an important result, it is even more significant to be able to accurately predict the storm track of a severe weather event well in advance. Reale et $\mathrm{a}^{14}$ examined the ability to predict in advance the storm track of Tropical Cyclone Nargis based on forecasts from the Control assimilation, the Radiance assimilation, and the AIRS Standard assimilation. The Quality Controlled AIRS temperature soundings used in that study were obtained from the Goddard DISC, and they, by definition, used the AIRS Standard Quality Control. Quality Controlled AIRS temperature profiles for this time period using Tight Quality Control were not available in time for that study.

Reale et $\mathrm{al}^{14}$ showed that Control analyses in the days prior to the landfall of Tropical Cyclone Nargis contained substantial misrepresentations, or even lack of representation, of the location a cyclone in the Bay of Bengal. Consequently, the storm track of this devastating storm was very poorly predicted ahead of time (as occurred in reality). The prior analyses and subsequent forecasts of the Nargis storm track were significantly better when AIRS Standard Quality Controlled temperature soundings were assimilated, and in fact an excellent prediction of when and where Nargis would hit land was produced from the AIRS Standard analysis 108 hours (4.5 days) ahead of forecast time. An intermediate ability to predict landfall of Nargis was produced using forecasts from the AIRS Radiance analysis.

It is apparent that assimilation of Quality Controlled AIRS temperature profiles can significantly improve 5-7 day forecast skill compared to that obtained without the benefit of AIRS data. In addition, assimilation of Quality Controlled 


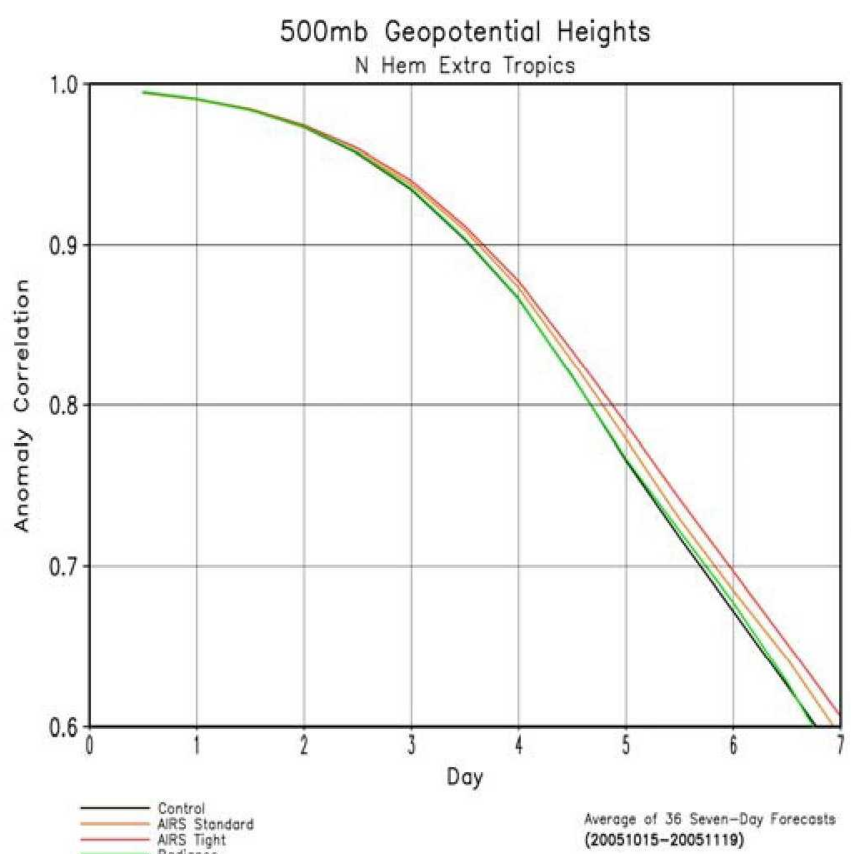

Figure 5a

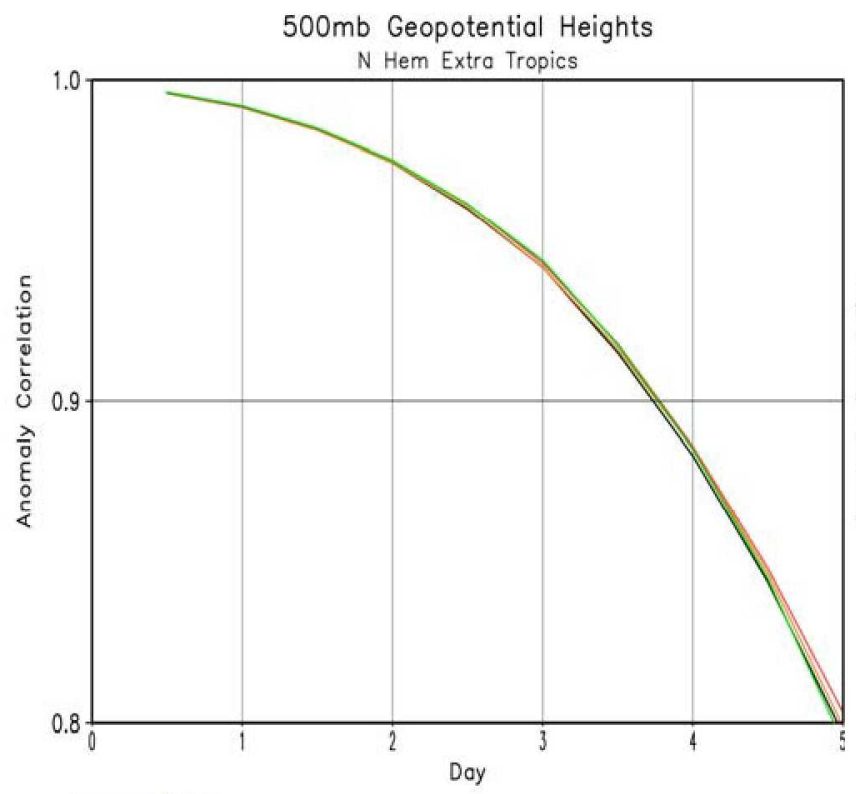

Contral

Averoge of 32 Forecosts (20080416-20080518)

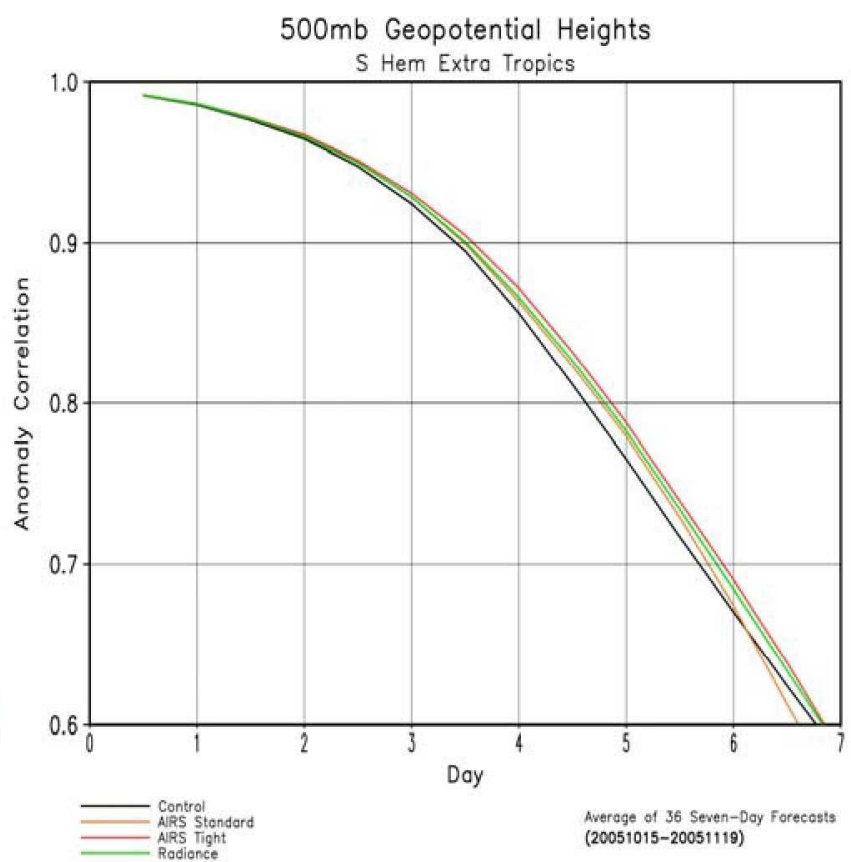

Figure 5b

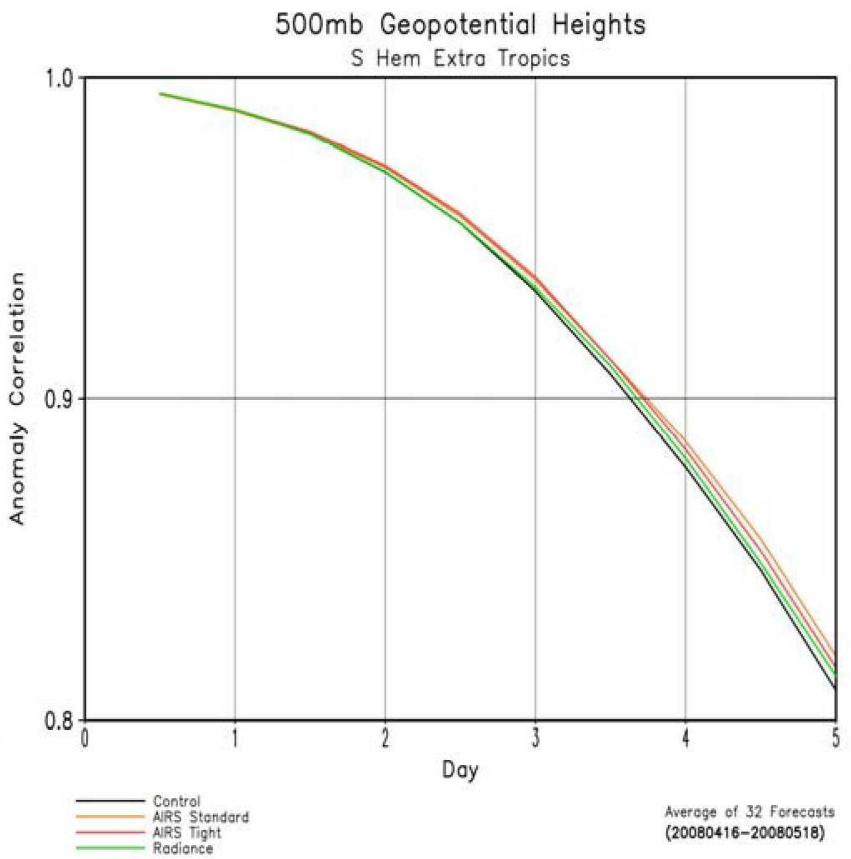

Figure 6b 
AIRS temperature soundings performs better than assimilation of AIRS observed radiances. Based on the experiments shown, use of the AIRS Tight Quality Control performs best on the average. We are conducting further studies to optimize the best trade between spatial coverage and accuracy for NWP purposes. We will also assess the assimilation of Quality Controlled values of AIRS clear column radiances $\hat{R}_{i}$ for NWP purposes

\section{SUMMARY}

Products derived using the AIRS Science Team Version 5 retrieval algorithm are generated operationally at the Goddard DISC. Version 5 contains accurate case-by-case error estimates for most derived products, which are also used for Quality Control. We have conducted forecast impact experiments in three different seasons and years assimilating AIRS Quality Controlled temperature profiles using the NASA GEOS-5 data assimilation system, consisting of the NCEP GSI analysis coupled with the NASA fvGCM. Assimilation of Quality Controlled temperature profiles resulted in significantly improved forecast skill in both the Northern Hemisphere and Southern Hemisphere Extra-Tropics, compared to these obtained from analyses obtained when all data used operationally by NCEP except for AIRS data was assimilated.

Experiments using different Quality Control thresholds for assimilation of AIRS temperature retrievals showed that a Tight Quality Control threshold performed better than the Standard threshold used in the AIRS Version 5 Quality Control procedure. Use of this Tight threshold provides better overall sounding accuracy than the Standard threshold but with somewhat poorer spatial coverage. We are conducting more experiments to further optimize this balance of spatial coverage and sounding accuracy from the data assimilation perspective.

In all cases, Quality Controlled AIRS temperature soundings were assimilated well below cloud level in partially cloudy cases. The positive impact of assimilating AIRS derived atmospheric temperatures all but vanished when only AIRS stratospheric temperatures were assimilated. Forecast skill resulting from assimilation of AIRS radiances uncontaminated by clouds, instead of AIRS temperature soundings, was not as good as that obtained by assimilation of the Quality Controlled temperature profiles. This reduction in forecast skill is most likely the result of significant loss of tropospheric information when only AIRS radiances unaffected by clouds are used in the data assimilation process.

\section{ACKNOWLEDGEMENT}

This work benefitted considerably from the collaboration with members of the OSE-OSSE team at the NASA GSFC Laboratory for Atmospheres and Software Integration and Visualization Office, who performed the data assimilation experiments, the results of which are shown in this paper. Their work was supported by Grant MAP/04-0180-0070 (P.I.: Oreste Reale).

\section{REFERENCES}

1. Pagano, T. S., H. H. Aumann, D. E. Hagan, and K. Overoye, "Prelaunch and in-flight radiometric calibration of the Atmospheric Infrared Sounder (AIRS)," IEEE Trans. Geosci. Remote Sensing, 41, 265-273, 2003.

2. Susskind, J., C. D. Barnet, and J. M. Blaisdell, "Retrieval of atmospheric and surface parameters from AIRS/AMSU/HSB data in the presence of clouds," IEEE Trans. Geosci. Remote Sensing, 41, 390-409, 2003

3. Susskind, J., C. Barnet, J. Blaisdell, L. Iredell, F. Keita, L. Kouvaris, G. Molnar, and M. Chahine, “Accuracy of geophysical parameters derived from Atmospheric Infrared Sounder/Advanced Microwave Sounding Unit as a function of fractional cloud cover," J. Geophys. Res., 111,D09S17, doi:10.1029/2005JD006272, 2006.

4. Susskind, J, Blaisdell, J. M., Iredell, L. and Keita, F., "Improved Temperature Sounding and Quality Control Methodology Using AIRS/AMSU Data: The AIRS Science Team Version 5 Retrieval Algorithm, IEEE Trans. Geosci. Remote Sensing, submitted 2009.

5. Tian, B., D. E. Waliser, E. J. Fetzer, B. H. Lambrigsten, Y. L. Yung, and B. Wang, "Vertical moist thermodynamic structure and spatial-temporal evolution of the MJO in AIRS observations", J. Atmos. Sci, 63, 2462-2485, 2006 
6. Le Marshall, J., et al., "Improving global analysis and forecasting with AIRS", Bull. Am. Meteorol. Soc, 87, 747-750, 2006.

7. Wu, L., S. A. Braun, J. J. Qu, and X. Hao, "Simulating the formation of Hurricane Isabel (2003) with AIRS data", Geophys, Res. Lett, 33, L04804, doi:10:1029/2005GL024665, 2006.

8. Wu, W.-S., R. J. Purser, and D. F. Parrish, "Three-dimensional variational analysis with spatially inhomogeneous covariances", Mon. Weather Rev., 130, 2905-2916, 2006.

9. Bosilovich, M. G., S. D. Schubert, M. Rienecker, R. Todling, M. Suarez, J. Bacmeister, R. Gelaro, G.-K. Kim, I. Stanjer, and J. Chan, "NASA's modern era retrospective-analysis for research and applications", U.S. CLIVAR Variations, 4, 5-8, 2007

10. Lin-S.J., "A 'vertically langrangian' finite-volume dynamical core for global models, Mon. Weather Rev, 132, 22932307, 2004.

11. Atlas, R., O. Reale, B.-W. Shen, S.-J. Lin, J.-D. Chern, W. Putman, T. Lee, K.-S. Yeh, M. Bosilovich, and J. Radakovich, "Hurricane forecasting with the high-resolution NASA finite volume general circulation model". Geophys. Res. Lett, 32, L03807, doi:10.1029/2004GL021513, 2005.

12. Shen, B.-W., R. Atlas, O. Reale, S.-J. Lin, J.-D. Chern, L. Chang, C. Henze, and J.L. Li, "Hurricane forecasts with a global mesoscale-resolving model: Preliminary results with Hurricane Katrina (2005)", Geophys, Res. Lett, 33, L13813, doi:10.1029/2006GL026143, 2006.

13. Reale, O., J. Susskind, R. Rosenberg, E. Brin, E. Liu, L. P. Riishojgaard, J. Terry, and J. C. Jusem, "Improving forecast skill by assimilation of quality-controlled AIRS temperature retrievals under partially cloudy conditions", Geophys. Res. Lett, 35, L08809, doi:10.1029/2007GL033002, 2008.

14. Reale, O., W. K. Lau, J. Susskind, E. Brin, E. Liu, L. P. Riishojgaard, M. Fuentes, and R. Rosenberg, "AIRS impact on the analysis and forecast track of tropical cyclone Nargis in a global data assimilation and forecasting system", Geophys. Res. Lett, 36, L06812, doi:10.1029/2008GL037122, 2009. 